\title{
Post Chickenpox Sequel in Children: Three Distinct Presentation
}

\author{
*T Khondaker ${ }^{1}$, SK Amin ${ }^{2}$, M Setu ${ }^{3}$, K Roy ${ }^{4}$ \\ ${ }^{1}$ Dr. Tarannum Khondaker, Registrar, Department of Pediatrics, AKMMC \\ ${ }^{2}$ Prof. Dr. Syed Khairul Amin, Professor \& Head, Department of Pediatrics, AKMMC \\ ${ }^{3}$ Dr. Mumtahina Setu, Assistant Professor, Department of Pediatrics, AKMMC \\ ${ }^{4}$ Dr. Kuntal Roy, Registrar, Department of Pediatrics, AKMMC \\ *Corresponding author
}

Date of submission: 10 September 2015 Date of acceptance: 23 March 2016

\begin{abstract}
Varicella (Chicken pox) is a common paediatric disease which usually self-limiting and resolves without sequelae. Complications are not common in immune-competent children. We present a series of three cases with rare complications following chicken pox, presented with distinct diagnoses, the etiology for which was established on the basis of history, examination and serological tests . The first two cases had neurological complications and the third case was a diagnosed case of hemophilia and presented with osteomylitis. All these complications developed following chicken pox. The cases responded differently to treatment and the patients were left with minimum disability.
\end{abstract}

Key Words: Post Chicken pox sequelae, Neurological complications, Osteomylities

\section{Introduction}

Varicella, commonly known as chickenpox, is caused by the varicella-zoster virus. The disease is generally regarded as a mild, self-limiting viral illness with occasional complications. Varicella is common and highly contagious and affects nearly all susceptible children before adolescence. Although most varicella infection confers life-long immunity, varicella clinical reinfections among healthy children are also found. ${ }^{1}$ Before varicella vaccination became widespread, 4 million cases of chickenpox were reported annually. The adoption of universal vaccination against varicella in 1995 reduced the incidence of varicella, as well as the associated morbidity and mortality rates. ${ }^{2}$ The causative organism, varicella-zoster virus, is a member of the human herpes virus subfamily Alphaherpesvirinae and, like all herpes viruses, is a DNA virus. The virus enters through the respiratory system (conjunctival or upper respiratory mucosa) and colonizes the upper respiratory tract. Viral replication takes place in regional lymph nodes over the next 2-4 days; 4-6 days later, a primary viremia spreads the virus to endothelial cells in the spleen, liver, and elsewhere. After a week, a secondary viremia disseminates the virus to the viscera and skin, eliciting the typical skin lesions. This viremia also spreads the virus to respiratory sites and is responsible for the contagion of varicella before the appearance of the rash. Infection of the central nervous system (CNS) or liver also occurs at this time, as may encephalitis, hepatitis, or pneumonia. The virus is spread in droplets of saliva through the air or by direct contact with the fluid from the blisters of the infected person. Coughing and sneezing are the most common modes of transmission, particularly in children. ${ }^{3}$

The usual incubation period is 10-21 days. The patient is contagious from 1-2 days before the appearance of rash until the lesions crust over, usually 5-6 days after the rash first appears .The nature of the infectious period makes it very difficult to prevent the disease from spreading. Varicella is associated with humoral and cellmediated immune responses. These responses induce long-lasting immunity. Repeat subclinical infection can occur in these persons, but second attacks of chickenpox are extremely rare in immune competent persons. Re-exposure and subclinical infections may serve to boost the immunity acquired after an episode of chickenpox. ${ }^{4}$ 


\section{Case 1}

A 10 years old afebrile boy presented with the complaints of intermittent, involuntary, nonrhythmic jerky movements of the right shoulder, which lasted few seconds and occasional diffuse headache, along with drowsiness for 7 days. He had a recent $\mathrm{H} / \mathrm{O}$ Chicken Pox 15 days back .He had no $\mathrm{H} / \mathrm{O}$ fall or taking any offending drugs that may causes the symptoms. He was drowsy without any signs of meningeal irritations and no neurological deficits are evident except jerky movements of the right shoulder. His cerebellar function was intact. His complete blood count showed normal findings with normal MRI of brain. CSF Study revealed 40 cells $/ \mathrm{ml}$; all were lymphocytes and protein was $65 \mathrm{mg} / \mathrm{dl}$. CSF glucose was $45 \mathrm{mg} / \mathrm{dl}$. After giving treatment with haloperidol and acyclovir child responded dramatically but developed dystonia due to extra pyramidal effects of haloperidol which resolved promptly with procyclidine hydrochloride.

\section{CASE 2}

A 8 year-old male presented with sudden onset weakness with unsteady gait that developed 10 days following chicken pox infection. He had dysarthia and drowsiness with mild vertigo. On examination, the skin showed multiple small scars and some with scabs. These lesions were mainly on chest wall, abdomen and few on the face. The examination of the nervous system revealed normal motor and sensory function except decreased coordination as evidenced by finger nose test and heel shin test with marked postural instability without any signs of meningeal irritations . No other cranial nerve abnormality was detected. Routine blood tests were normal. Computed tomography (CT) scan of brain was normal. Cerebrospinal fluid (CSF) examination showed 25 cells $/ \mathrm{ml}$; all were lymphocytes and protein was $65 \mathrm{mg} / \mathrm{dl}$. CSF glucose was $70 \mathrm{mg} / \mathrm{dl}$ (simultaneous blood glucose $130 \mathrm{mg} / \mathrm{dl}$ ). The CSF showed increased titer of IgM anti-varicella zoster virus (anti-VZV) antibody (1:256). For CSF, any titer is considered significant.The patient responded to acyclovir and prednisolone. The prednisolone was continued for a period of 2 weeks and then tapered. The acyclovir was continued for 10 days. The cerebellar symptoms took one month to resolve fully.

\section{Case 3}

A 14 years old boy with haemophilia B presented with pain,swelling and redness below the knee joint without any history of trauma, which caused him difficulty to bear weight on his right leg. He had varicella two weeks prior to this illness. Conventional X-ray showed joint destruction affecting the metaphyseal bone of distal right femur and common periosteal reaction. These findings were consistent with osteomyelitis and the patient was operated by an orthopedic physician. Intraoperatively, massive necrotic debris and seropurulent fluid were found around the periosteum of femar and was removed. Complete blood count showed abundant polymorphonuclear leucocytes and cultures were negative. Intravenous treatment with Ceftriaxone and flucloxacillin were started and continued for 2 weeks along with factor IX. The patient was discharged and oral flucloxacillin and cefradin were administered for a further 2 months. Follow up X-ray analysis showed improved radiological findings, thereby indicating successful recovery.

\section{Discussion}

Though varicella is a self limiting benign disease, occasionally it may cause complications. The cause of complications has been postulated as either direct viral invasion or through an immune-mediated allergic mechanism. Most pathologic studies have shown a picture more likely to be allergy-mediated injury. ${ }^{5}$

There was a seasonal distribution of reported complications with a peak in March. The majority of complications occurred in preschool-age children with a maximum age of 4 years. No gender predominance was found with a distribution. Multiple entries for complications were allowed. The most frequent complications were neurological. Among all infectious complications superinfections of the skin, pyogenic arthritis, osteomyelitis, necrotizing fasciitis, orbital cellulitis and pneumonia were also identified. Infectious complications were reported in the majority in younger children up to 4 years of age, whereas neurologic complications occurred more frequently in an older age range. ${ }^{6}$

Although Herpes zoster (HZ) is considered a disease of the elderly, it can affect individuals at any age ,including children and adolescents associated with immunosuppressive conditions such as malignancy, especially leukemia and HIV infection. It has also been reported in otherwise healthy children, and 
most frequently in those with varicella infection in the first year of life. ${ }^{7}$

The musculoskeletal complications that were observed were cellulitis, pyomyositis, osteomyelitis and gangrene. Osteomyelitis should always be considered in any child who develops pain, swelling, and limitation of movement in a limb during or after varicella infection. A review of literature shows 12 reported cases of osteomylities where Group B haemolytic streptococcus were isolated from blood culture and skin lesion. ${ }^{8}$ About $5 \%$ of children with varicella develop otitis media, caused by the usual pathogens. Hepatitis is a selflimited accompaniment of varicella. Retinitis and optic neuritis have been reported as rare complications of varicella in children who are immunocompetent. ${ }^{9}$ Other reported complications include glomerulonephritis, hemorrhagic varicella, thrombocytopenia, myocarditis, appendicitis, pancreatitis, Henoch-Schönleinpurpura, orchitis, iritis, and keratitis. ${ }^{10}$

Common CNS complications of chicken pox are cerebellar ataxia and encephalitis. The commonest presentation is encephalitis. In one series by Miller et al, encephalitis accounted for $90 \%$ cases and $37 \%$ of these had cerebellar involvement. Rare complications include transverse myelitis, aseptic meningitis, Guillian-Barre syndrome, meningoencephalitis, ventriculitis, optic neuritis, post-herpetic neuralgia, herpes zoster ophthalmicus, delayed contralateral hemiparesis, peripheral motor neuropathy, cerebral angitis, Reye syndrome and facial paralysis. Complications are generally mild, with good prognosis and very low mortality. The virus is known to cause vasculitis-like episodes and even cerebrovascular accidents have been reported. In immunocompromised host, the virus can invade deeper tissues and the virus has been isolated from brain tissue or ventricles by polymerase chain reaction (PCR). The CSF usually reveals a mononuclear pleocytosis and oligoclonal bands. Cerebral angiography also reveals areas of focal arterial stenosis or occlusion. Macroscopically, a predominance of gray-white matter junction lesions is seen. Microscopically, the virus is present in affected cerebral arteries,but not in areas of infarction, although in chronic cases virus may be seen in brain parenchyma, usually close to arteries and veins. The primary site of VZV is in cerebral arteries which contain multinucleated giant cells, Cowdry A inclusion bodies, and herpes virus particles. Tentorial and dural enhancement is reported in a few cases of chicken pox. It can be caused by immune-mediated Acute Disseminated Encephalomyelitis [ADEM] or by meningoencephalitis. ${ }^{5}$

Radiculopathy in isolation after chicken pox is extremely rare and a high degree of clinical suspicion is needed to identify it. Various cases with various pathogenesis mechanisms have been reported since the first case was reported in 1924. Depending on the pattern of involvement (axonal, sensory or autonomic), patients generally improve over several days. Treatment is with IV Ig or plasmapheresis and ventilator support, if the need arises. ${ }^{11}$ The disease usually lasts for 2-4 weeks, but can be as short as 3 days or as long as several months. ${ }^{12}$

Acute transverse myelitis (ATM) can be occur following chicken pox. Treatment of ATM is with corticosteroids, and methylprednisolone intravenous has been found to be effective in one study. Antivirals have a controversial role and physiotherapy has a definitive supportive and rehabilitative role, including in those with bladder involvement. ${ }^{13}$

The most common infectious organisms are group A Streptococci and Staphylococcus aureus. In addition to toxic shock syndrome, group A streptococci may cause necrotizing fasciitis, bacteremia, osteomyelitis, pyomyositis, gangrene, subgaleal abscess, arthritis, and meningitis in patients with varicella. Staphylococci reportedly cause cellulitis, impetiginous pox infections, staphylococcal scalded skin syndrome, toxic shock syndrome, pericarditis, and osteomyelitis in these patients. ${ }^{14}$

In general, laboratory studies are unnecessary for diagnosis, because varicella is clinically obvious. However, some tests and procedures may be helpful in confirming the diagnosis or identifying complications. Imaging studies are typically not required for varicella unless secondary complications are a concern. Most children with varicella have leukopenia in the first 3 days, followed by leukocytosis. Marked leukocytosis may indicate a secondary bacterial infection but is not a dependable sign. Significant elevations of alanine aminotransferase (ALT) occur in $20-50 \%$ of children and adolescents with varicella complicated by hepatitis, but elevations return to normal within one month in almost all cases. Tzanck smear involves scraping the base of the lesions and then 
staining the scrapings to demonstrate multinucleated giant cells. The presence of multinucleated giant cells suggests a herpes virus infection but is not specific for varicella-zoster virus. Immuno histochemical staining of skin lesion scrapings can confirm varicella. The procedure is useful for highrisk patients who require rapid confirmation. Serology is mainly used to confirm past infection to assess a patient's susceptibility status. This helps determine preventive treatment requirements for an adolescent or adult who has been exposed to varicella. Among the many serologic studies, the most sensitive are the indirect fluorescent antibody (IFA), fluorescent antibody to membrane antigen (FAMA), neutralization test (NT), and radioimmunoassay (RIA). Commercially available latex agglutination (LA) and enzyme-linked immunosorbent assay (ELISA) tests are sensitive and rapid. Polymerase chain reaction (PCR) examination of skin scrapings is quick and sensitive.

This test is replacing other methods as the equipment becomes more widely available. Bacterial culture of lesions may be indicated if signs of superinfection are present.Chest radiographic findings may be normal or may show diffuse bilateral nodular infiltrates in primary varicella pneumonia. Radiography may also detect focal infiltrates suggestive of secondary bacterial pneumonia. Children with neurological signs should have their cerebrospinal fluid (CSF) examined. The CSF of patients with varicella encephalitis may have few or as many as 100 cells that are polymorphonuclear or mononuclear, depending on the timing of the lumbar puncture. Glucose levels are within the reference range. Protein levels are within the reference range or are slightly elevated. ${ }^{15}$

Treatment approaches include supportive measures, antiviral therapy, administration of varicella zoster immune globulin (VZIG), and management of secondary bacterial infection.

One should isolate patients with varicella because the disease is highly contagious and airborne spread can occur. Isolation is especially important if the hospital also admits patients who are immunocompromised because their exposure to the disease can be serious and even fatal.

The routine use of acyclovir or valacyclovir in healthy children is recommended by the AAP (American Academy of Paediatrics) if it can be given within 24 hours after the rash first appears in children older than 12 years, in immunocompromised children or in healthy children with varicella pneumonia or encephalitis. In some instances, acyclovir may be considered for teenagers and adults with otherwise uncomplicated varicella. Additionally, antiviral therapy should be considered for patients with recent steroid use or those with extensive eczema.Varicella zoster immune globulin (VariZIG by Cangene) was approved by the FDA in December 2012. It is indicated for high-risk individuals within 10 days (ideally within 4 days) of chickenpox exposure. This agent reduces complications and the mortality rate of varicella, not its incidence. Intravenous immunoglobulin (IVIG) has been used to prevent varicella after exposure when VZIG is not available. ${ }^{16}$

Varicella vaccine consists of live attenuated Oka strain varicella virus. The vaccine is safe and highly immunogenic. It was approved for use in the United States in 1995 and has greatly reduced the incidence and mortality due to varicella. The vaccine has a protective efficacy of $71-100 \%$ against varicella. However, it affords a much greater degree of protection against moderate and severe varicella $(95-100 \%) .{ }^{17}$

Babies are born with protective maternal antibodies to varicella. The half-life of these antibodies is about 6 weeks, and most children have very low levels beyond age 5 months. However, the varicella vaccine is recommended after age 1 year. A single dose provides protection to approximately $85 \%$ of recipients. ${ }^{18}$ The Advisory Committee on Immunization Practices (ACIP) and the American Academy of Pediatrics (AAP) now recommend 2 doses of this vaccine for all children. After the first dose at age 12-15 months, the second should be administered at age 4-6 years. Two doses of the vaccine provide $98 \%$ protection against varicella and $100 \%$ protection against severe disease.These children also have a lower incidence of breakthrough varicella.Breakthrough disease involves varicella that occurs after 42 days of immunization. When it occurs, it is usually mild disease but can spread to other susceptible individuals. ${ }^{19}$

Postexposure prophylaxis with the vaccine, if provided within 36-72 hours of contact, can prevent or attenuate disease in the exposed individual. Although vaccinated children develop milder disease, they are still infectious. ${ }^{20}$

It is estimated that there is a slightly higher risk of febrile seizures in children aged 12-23 months vaccinated with the MMRV when compared with separate MMR and varicella vaccine administration. ${ }^{21}$ 


\section{Conclusion}

Complications of varicella requiring hospitalization in immunocompetent children are more frequent than previously thought. Varicella surveillance is needed to facilitate public health action at the state and local level and to monitor the impact of the varicella immunization program. The implementation of an effective varicella vaccine program for healthy children would be beneficial for the prevention of complications and the reduction in their associated cost to the health care system.

\section{Conflicts of Interest}

The authors have no conflict of interest to anybody.

\section{Reference}

1. Hall S, Maupin T, Seward J, Jumaan AO, Peterson C, Goldman G, et al. Second varicella infections: are they more common than previously thought? Pediatrics. 2002; 109(6): 1068-73.

2. Shah SS, Wood SM, Luan X, Ratner AJ. Decline in varicella-related ambulatory visits and hospitalizations in the United States since routine immunization against varicella. Pediatr Infect Dis J. 2010; 29(3): 199-204.

3. The Paediatric Society of New Zealand and Starship Foundation. Auckland. KidsHealth (2012) Chickenpox. http:// www.kidshealth.org.nz/ chickenpox

4. Rockley PF, Tyring SK. Pathophysiology and clinical manifestations of varicella zoster virus infections. Int J Dermatol. 1994; 33(4): 227-32.

5. PaulR, Singhaniap, HashmiMA,Bandyopadhyay R, and Banerjee A K. Post chicken pox neurological sequelae: Three distinct presentations. J Neurosci Rural Pract. 2010; 1(2): 92-96

6. ZieboldC, KriesR V, Lang R, Weigl J. Severe Complications of Varicella in Previously Healthy Children in Germany: A 1-Year Survey Pediatrics 2001. 108( 5), pp. e79

7. Epidemiology of Pediatric HerpesZoster After Varicella Infection:A Population-Based Study. SuYing Wen S Y, Liu W L,February 23, 2015(doi: $\begin{array}{llllllllllllllllllll} & 0 & 1 & 5 & 4 & 2 & / & \mathrm{p} & \mathrm{e} & \mathrm{d} & \mathrm{s} & . & 0 & 1 & 3 & -\end{array}$ 4037).pediatrics.aappublications.org/content/early/ .../peds. 2013-4037.

8. Gupta B, Anil K, Abdullah A. Varicella-related musculoskeletal complications in children . Journal of Pediatric Orthopaedics B: 2011; 20 (4 ): 264269
9. Tappeiner C, Aebi C, Garweg JG. Retinitis and optic neuritis in a child with chickenpox: case report and review of literature. Pediatr Infect Dis J. 2010. 29(12): 1150-2

10. Matsukura H, Murakami M, Sakaki H, Mitani T, Shimura S. Varicella glomerulonephritis preceding the cutaneous lesions. ClinNephrol. 2009 . 72(2): 161-2

11. Madan S, Aneja S, Tripathi RP, Batra A, Seth A, Taluja V. Acute disseminated encephalomyelitis: A case series. Indian Pediatr. 2005; 42(1): 367-71

12. Gnann JW. Varicella-Zoster virus: Atypical presentations and unusual complications. J Infect Dis. 2002; 186(4): 91-8.

13. La Rovere KL, Raju GP, Gorman MP. Post varicella acute transverse myelitis in a previously vaccinated child. Paedaitr Neurol. 2008; 38(5): 370-2.

14. Lesko SM, O'Brien KL, Schwartz B, Vezina R, Mitchell AA. Invasive group A streptococcal infection and nonsteroidal antiinflammatory drug use among children with primary varicella. Pediatrics. 2001; 107(5): 1108-15

15. Kristen A B.Paediatric chickenpox: Backgroumd, pathophysiology and etiology. emedicine. medscape.com/ article/ 969773. May 2015.

16. American Academy of Pediatrics Committee on Infectious Diseases: The use of oral acyclovir in otherwise healthy children with varicella. Pediatrics. 1993 Mar. 91(3): 674-6.

17. Marin M, Meissner HC, Seward JF. Varicella prevention in the United States: a review of successes and challenges. Pediatrics. 2008 Sep. 122(3): e744-51

18. Pinquier D, Gagneur A, BaluL .Prevalence of antivaricella-zoster virus antibodies in French infants under 15 months of age.Clin Vaccine Immunol. 2009; 16(4): 484-7

19. Lee LE, Ho H, Lorber E. Vaccine-era varicella epidemiology and vaccine effectiveness in a public elementary school population, 2002-2007. Pediatrics. 2008; 121(6): 1548-54

20. Quian R J, Protasio P A, Dall'orso V P. [Varicella outbreak in a village in Uruguay]. Rev ChilenaInfectol. 2010 Feb. 27(1): 47-51.

21. American Academy of Pediatrics Committee on Infectious Diseases. Policy Statement--Prevention of Varicella: Update of Recommendations for Use of Quadrivalent and Monovalent Varicella Vaccines in Children. Pediatrics. 2011 Aug 28. 


\section{Case Report}

An 18 years old phenotypically female presented with the complaints of swelling in both groin since birth, non-establishment of menstruation and generalized weakness for last 6th months. The swelling in both inguinal regions were gradually increasing in size and painless. She was immunized according to EPI schedule. She took some homeopathic drugs for the swelling.

Clinical examination revealed the swellings were oval in shape $4 \times 3 \mathrm{~cm}$ in left side and $3 \times 2 \mathrm{~cm}$ in right side, surface smooth, well defined margin, soft in consistency, mobile from side to side $\&$ above downwards, free from overlying skin \& underlying structure. There was no visible pulsation, no local rise of temperature, nontender but reducible by patient herself. The swellings disappeared on lying position \& appeared on coughing \& sitting. There was no axillary or pubic hair. Breasts were under developed with pale areola. Labia minora \& clitoris was present with blind vagina.

The hormonal levels were the following (Table 1). USG of pelvic organs revealed absent uterus \& its appendages suggests bilateral Müllerian developmental arrest, gonads were separate \& appears like undescended testis located in deep inguinal rings of either side. The cytogenetic karyotype results in 46XY abnormal male with gonadal dysgenesis.

Other laboratory investigations including complete blood count, random blood sugar, chest $\mathrm{x}$ ray, liver function test were normal. After communicating the results to the parents we decided to perform open gonadectomy under general anesthesia. (Which confirmed the results described by ultrasound). Considering the high incidence of malignant degeneration of the gonads held in the abdominal cavity, we addressed the patient to bilateral gonadectomy.

Table I: Hormonal Levels

\begin{tabular}{ll}
\hline Analyte & Value \\
\hline FSH & $60.66 \mathrm{mLU} / \mathrm{mL}$ \\
LH & $24.73 \mathrm{mLU} / \mathrm{mL}$ \\
Progesterone & $0.88 \mathrm{ng} / \mathrm{ml}$ \\
Prolactin & $477.40 \mathrm{mLU} / \mathrm{L}$ \\
Testosterone & $84.23 \mathrm{ng} / \mathrm{mL}$ \\
T3 & $2.23 \mathrm{ng} / \mathrm{mL}$ \\
T4 & $8.89 \mu \mathrm{g} / \mathrm{dL}$ \\
TSH & $3.32 \mu \mathrm{Ul} / \mathrm{mL}$ \\
\hline
\end{tabular}

Histological examination of biopsy tissue from inguinal swellings showed testicular parenchyma, seminiferous tubules of smaller in size than the normal, and agenesis of the germinal epithelium.

\section{Discussion}

Clinical features of this syndrome include a totally female aspect from birth onwards. One important feature that helps to address correct diagnosis is that at puberty breast develops regularly, while there is scarce or absent development of pubic and axillary hair ${ }^{7}$. Diagnosis of CAIS could be very early, for example, when the mother underwent an amniocentesis that reported a 46XY karyotype, and on the contrary obstetrics ultrasound or clinical evidence at birth showed the presence of female external genitalia ${ }^{9,10}$. Another clinical element that could address the diagnosis of CAIS is the development of unilateral or bilateral inguinal hernia in the apparently female patient ${ }^{11}$. Patients with PAIS show very different clinical phenotype that depends on the severity of undervirilization. Endocrine features of CAIS and PAIS are the same: we could observe normal or overproduced serum Luteinizing Hormone ( $\mathrm{LH}$ ) and Testosterone (T) during the first three months of life. After this, LH and $T$ levels are in the normal range until the puberty $^{12}$. Then, at the puberty, we will find elevated serum levels of $\mathrm{T}$ and of $\mathrm{LH}$, due to the androgen insensitivity and the consequent lack of negative feedback exerted by sex hormone on hypothalamus and hypophysis. Testosterone becomes itself target of aromatase, and so this enzyme covert it into estrogens: for this reason, CAIS patients present with higher estrogens levels than normal male and have good development of breast. Moreover, in patients with AIS, AntiMüllerian Hormone (AMH) concentration is normal as the secretion and function of Sertoli and Leydig cells are not impaired ${ }^{13}$. In the following we will describe a case of CAIS.

\section{Conclusion}

All these findings were consistent with Complete Androgen Insensitivity Syndrome. Therefore, the 
patient underwent a surgery for removal of gonads and was referred to hormone replacement therapy(HRT). Parents preferred not to disclose the diagnosis to their daughter. Hormone therapy was needed but could resolve neither amenorrhea nor infertility due to absence of uterus and ovaries besides there is every possibility of developing adverse effects of long term HRT. Later the patient could be offered vaginoplasty to alleviate her psychosocial embarrassment. This is a very devastating news for a family where a young psychologically female needs adequate familial and social support to lead a near normal life.

\section{Conflict of interest: none}

\section{References}

1. Ahmed SF, ChengA, Hughes IA. "Assessment of the gonadotrophin-gonadal axis in androgen insensitivity syndrome". Arch. Dis. (April 1999) Child. 80 (4): 324-9.

2. Jones RE, Lopez KH "Chapter 5: Sexual differentiation". Human reproductive biology. Amsterdam: Elsevier Academic Press. (2006). pp. 127-148. ISBN 0-12-088465-8.

3. EL, Loy CJ, Sim KS "Androgen receptor gene and male infertility". Hum. Reprod. Update. (2003). 9 (1):17 doi:10.1093/humupd /dmg003PMID 12638777.

4. Sobel V, Schwartz B, Zhu YS, Cordero JJ, Imperato-McGinley J. "Bone mineral density in the complete androgen insensitivity and 5 alphareductase-2 deficiency syndromes". J. Clin. Endocrinol. Metab(August 2006).91 (8):3017-23. doi:10./ jc.20052809. PMID 16735493.

5. C. A. Quigley, A. De Bellis, K. B. Marschke, M. K. El-Awady, E. M. Wilson, and F. S. French, "Androgen receptor defects: historical, clinical, and molecular perspectives," Endocrine Reviews, 1995; 16 (3): 271-321.
6. A. Ulloa-Aguirre, J. P. Mendez, B. Chavez, S. Carranza-Lira, A. Angeles, and G. PerezPalacios, "Incomplete regression of müllerian ducts in the androgen insensitivity syndrome," Fertility and Sterility, 1990; 53(6):1024-1028.

7. S. T. Dodge, M. S. Finkelston, and K. Miyazawa, "Testicular feminization with incomplete müllerian regression," Fertility and Sterility, 1985; 43(6): 937-938.

8. M. L. Swanson and E. H. Coronel, "Complete androgen insensitivity with persistent müllerian structures. A case report," The Journal of Reproductive Medicine,1993; 38(7): 565-568.

9. J. D. Stephens, "Prenatal diagnosis of testicular feminisation," The Lancet, 1984; 2(8410): 1038.

10. O. Hiort, Q. Huang, G. H. G. Sinnecker et al., "Single strand conformation polymorphism analysis of androgen receptor gene mutations in patients with androgen insensitivity syndromes: application for diagnosis, genetic counseling, and therapy," Journal of Clinical Endocrinology and Metabolism, 1993; 77(1): 262-266.

11. M. M. Grumbach and F. A. Conte, "Disorders of sex differentiation," in Williams Textbook of Endocrinology, J. D. Wilson and D. W. Foster, Eds. 1991, pp. 853-951, Saunders, Philadelphia, $\mathrm{Pa}$, USA.

12. S. Campo, M. Stivel, and G. Nicolau, "Testicular function in post pubertal male pseudohermaphroditism," Clinical Endocrinology, 1979; 11(5): 481-490.

13. A. Galani, S. Kitsiou-Tzeli, C. Sofokleous, E. Kanavakis, and A. Kalpini-Mavrou, "Androgen insensitivity syndrome: clinical features and molecular defects," Hormones, 2008; 7(3):217-229. 